\title{
Signaling Strategy in Spermatozoa Activation of Sea Urchin, C. elegans and Human: Three Different Players for the Same Melody
}

Nicola Bernabò*, Ilaria Saponaro, Mauro Mattioli and Barbara Barboni

Department of Comparative Biomedical Sciences, University of Teramo, Teramo, Italy

\begin{abstract}
Male gametes become able to fertilize only after a series of chemical-physical modifications, the spermatozoa activation. The signaling strategy of this process, in organisms belonging to different Phyla and displaying completely different reproductive and ecological features (sea urchin, C. elegans and Human), was compared using a computational approach. To this aim the biological networks, i.e. networks of nodes (the molecules) linked each other by edges (their interactions) representing spermatozoa activation in these three species, were realized. Their statistical analysis revealed that: 1) the networks displayed a scale free topology, as expressed by the power-law degree distribution of the number of links per node; 2 ) the clustering coefficient, i.e. the measure of how each network node tend to cluster to other ones, was low, ranging from 0.023 to $0.032 ; 3$ ) the characteristic path length (the measure of how many links it is necessary to pass through to travel between two nodes) was comprised between 6.6 and $8.1 ; 4)$ a high percentage of nodes showed two links; 5) the most linked node, in all networks, was [Ca $\left.\left.{ }^{2+}\right] \mathrm{i}, 6\right)\left[\mathrm{Ca}^{2+}\right] \mathrm{i}$, glycolysis and mitochondrial oxidative phosphorylation were shared by all the examined organisms. Thus it is possible to conclude that spermatozoa activation in sea urchin, C. elegans and Human has similar signaling strategy and metabolism architecture, that could be due to the maintenance of ancestral mechanisms, and that confer to this process some important biological features such as robustness against random failure and signaling fastness, rapidity and efficiency.
\end{abstract}

Keywords: Human; C. elegans; Sea urchin; Spermatozoa; Sperm activation; Bioinformatics; Biological networks; Computational model

\section{Introduction}

Many cellular systems undergo a process of activation during their metabolic activity. For instance the contraction of striated muscle, the release of synaptosomes from the presynaptic endplate, the degranulation (exocytosis) of sensitized mast cells, the activation of mammals eggs, the activation of visual transmission in mammalian rods, the activation of male gametes to reach the full fertilizing ability require an activation process. This last event, in particular, has attracted the attention of Researches since its discovery in 50's because of its important implications both for basic (developmental biology, endocrinology, biochemistry) and applied science (andrology, male infertility, contraception). Thus sperm activation has been studied in several organisms, of different Phyla, and the data about this important topic are growing day by day. The availability of bioinformatics tools has provided to the Scientists new resources to investigate this phenomenon in its complexity. Overcoming the reductionist approach until now used, the use of bioinformatics resources makes possible the aggregation of molecular data in explicative models. The use of computational modeling to explore the biochemical architecture of mammalian sperm activation, thus allowing the quantitative analysis of this complex signaling system, has been recently introduced [1] and validated [2] by our group. In this context it could be very interesting to modelize and compare the sperm activation process in organisms showing completely different ecology, reproductive strategy and sexual behavior, as it is the case of sea urchin, C. elegans and Human. Sea urchins are members of the Phylum Echinodermata. They are dioecious, having separate male and female sexes, and have five gonads (fivefold symmetry) with a single duct, rising from the upper pole to open at a gonopore lying in one of the genital plates surrounding the anus [3]. The gonads are lined with muscles underneath the peritoneum, that allow the animal to squeeze its gametes through the duct and into the surrounding sea water, where fertilization takes place [3]. Sea urchin spermatozoa acquire the motility only once they reach sea water [4]. To increase the success rate of fertilization the male gametes are attracted by the molecules dispersed by the homologous oocytes. The outer investment of oocytes, the egg jelly, contains short sperm-activating peptides (SAPs) that bind to specific sperm receptors and dramatically alter the metabolic rate and motility of spermatozoa, driving them towards the oocytes [5]. When sperm encounter the egg jelly, the exocytosis of the acrosomal vesicle, the acrosome reaction (AR), occurs and the pHi-dependent polymerization of actin leads to the extension of acrosomal tubule, which exposes a new bindincovered membrane which will fuse specifically through a receptor to the egg [6,7]. The nematode C. elegans is an unsegmented, $1 \mathrm{~mm}$ long, vermiform and bilaterally symmetrical worm, living in the ground, having hermaphrodites and males. In particular the $99.95 \%$ of the individuals are hermaphrodite and the $0.05 \%$ of the population is composed by males. Males have a single-lobed gonad, a vas deferens, and a tail specialized for mating [8]. Hermaphrodites have two ovaries, an oviduct, a spermatheca (a kind of chamber where oocytes are fertilized by sperm), and a single uterus. C. elegans eggs are laid by the hermaphrodite and, after hatching, they pass through four juvenile stages (L1-L4). Hermaphrodites produce all their sperm in the L4 stage (150 sperm per gonadal arm), then they switch over to produce oocytes. The sperm are stored in the same area of the gonad as the oocytes until the first oocyte pushes the sperm into the spermatheca. The male can inseminate the hermaphrodite, which will preferentially use male

*Corresponding author: Nicola Bernabò, DMV, PhD, Department of Comparative Biomedical Sciences, Faculty of Veterinary Medicine, Piazza Aldo Moro 45, 64100 Teramo, Italy; Tel: +39 0861 266879; E-mail: nbernabo@unite.it

Received October 18, 2011; Accepted November 15, 2011; Published Novembe 18, 2011

Citation: Bernabò N, Saponaro I, Mattioli M, Barboni B (2012) TSignaling Strategy in Spermatozoa Activation of Sea Urchin, C. elegans and Human: Three Different Players for the Same Melody. J Bioengineer \& Biomedical Sci S5:006. doi:10.4172/2155-9538.S5-006

Copyright: (c) 2012 Bernabò N, et al. This is an open-access article distributed under the terms of the Creative Commons Attribution License, which permits unrestricted use, distribution, and reproduction in any medium, provided the original author and source are credited. 
Citation: Bernabò N, Saponaro I, Mattioli M, Barboni B (2012) TSignaling Strategy in Spermatozoa Activation of Sea Urchin, C. elegans and Human: Three Different Players for the Same Melody. J Bioengineer \& Biomedical Sci S5:006. doi:10.4172/2155-9538.S5-006

Page 2 of 6

sperm (both types of sperm are stored in the spermatheca). When self-inseminated C. elegans will lay approximately 300 eggs, on the contrary when inseminated by a male, the number of eggs can exceed 1.000 [8]. C. elegans spermatozoa are characterized by the absence of the flagellum and the lack of the acrosome, while they content many membranous vesicles, the Membranous Organelles (MOs). The motility is acquired by a process (the spermiogenesis or sperm activation), in which the spherical spermatid extends a pseudopod, conferring to the cell an amoeboid motility. This event is made possible by an important reorganization of the cytoskeleton (the C. elegans spermatozoa content MSP instead of actin) and of membrane involving the remodeling of specific microdomains [9].

In Human the reproduction is obtained by internal fertilization and the spermatozoa were ejaculated in vagina. Male gametes travel along the female genital tract until they reach the oviduct, where they reside for a relatively long period (from hours to days) thus acquiring the fertilizing ability. This process, called "the capacitation", involves virtually all the cellular structures: the intracellular calcium concentration rises $[10,11]$, the protein phosphorylation pattern changes [12,13], the actin cytoskeleton reorganizes [14], the plasma membrane and the outer acrosome membrane become more instable and gradually acquire the ability to fuse each other $[15,16]$, the spermatozoa motility is hyperactivated [17]).

Aim of the present work was to carry out a comparison among the different biochemical events occurring during sperm activation in these organisms, adopting the graph theory formalism. The molecules that interact each other during sperm activation in sea urchin, $C$. elegans and Human were represented by a mathematical object called "graph" [18], constituted by a variable number of nodes (the molecules) linked by edges (the interactions) and originating a network, thus, the statistical analysis of the main topological parameters of the networks was carried out.

\section{Materials and Methods}

\section{Database realization, network construction}

The data about human sperm activation were obtained from the already realized database [1] after updating. Since at present a database containing the data about sperm activation of sea urchin (the available data are in particular referred to S. purpuratus,) and C. elegans does not exist, a new database was realized using Microsoft Office Excel 2003. The available information was obtained from peer-reviewed papers from PubMed (www.ncbi.nlm.nih.gov/pubmed/). As a reference were used the data published during latest 10 years. In some cases the record did not represent a single molecule but complex events, such as "membrane fusion" or "protein tyrosine phosphorylation" because all the single molecular determinants of the phenomenon are still unknown.

The database fields were:

Source molecule: representing the molecule source of interaction.

Interaction: representing the nature of interaction (activation, inhibition).

Target molecule: representing the molecule target of the interaction.

Biological function: representing the functional meaning or the contest of interaction (glycolysis, lipid remodelling, oxidative phosphorylation).
Species: representing the species in which the interaction was described.

Reference: representing the bibliographic source of information.

Notes: representing all the notation such as the presence of synonyms or the intracellular location, if relevant, or the explanation of complex cellular events.

The data, extracted from the databases, were used to build the networks using the Cytoscape 2.6.3 software (www.cytoscape.org). The network representing the elements common to the three networks was realized, using the Cytoscape plugin Network Analyzer (function "Compute intersection" of "Compare two networks" menu). This new network was called CE (Common Elements) network.

The networks were spatially represented using the Cytoscape Degree Sorted Circle Layout: all nodes with the same number of links are located together around the circle (Cytoscape User Manual, http:// www.cytoscape.org/manual/Cytoscape2_6Manual.html). The node size was proportional to the number of connection and the node color gradient was dependent on the closeness centrality. This parameter is computed as: $\operatorname{Cc}(n)=1 / \operatorname{avg}(\mathrm{L}(n, m))$, where $\mathrm{L}(\mathrm{n}, \mathrm{m})$ is the length of the shortest path between two nodes $\mathrm{n}$ and $\mathrm{m}$. The closeness centrality of each node ranges from 0 to 1 and it is a measure of how fast information spreads from a given node to the other nodes.

\section{Data analysis}

The statistical and topological analyses of networks were carried out, in agreement with Bernabò [1,2] considering the networks as directed by the Cytoscape plugin Network Analyzer (www.med.bioinf mpi inf.mpg.de/netanalyzer/help/2.6.1/index.html).

\section{Results}

The networks representing spermatozoa activation of sea urchin, $C$.

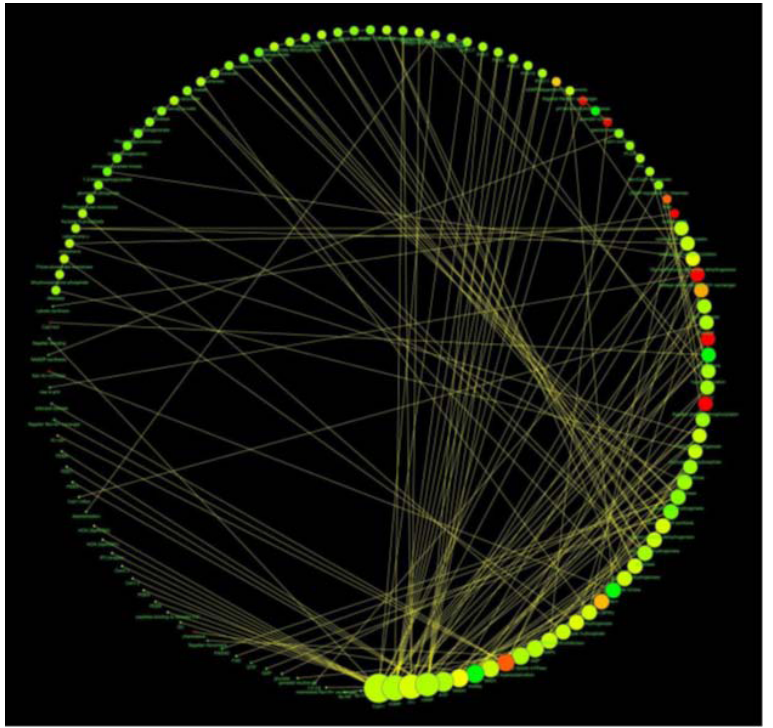

The nodes diameter is proportional to the number of links, the color varies depending on the closeness centrality (see text for explanation). The networks were spatially represented using the Cytoscape Degree Sorted Circle Layout: all nodes woth the same number of links are located together around the circle (see Cytoscape's User Manual).

Figure 1: Diagram showing the sea urchin spermatozoa activation network. 
Citation: Bernabò N, Saponaro I, Mattioli M, Barboni B (2012) TSignaling Strategy in Spermatozoa Activation of Sea Urchin, C. elegans and Human: Three Different Players for the Same Melody. J Bioengineer \& Biomedical Sci S5:006. doi:10.4172/2155-9538.S5-006

Page 3 of 6

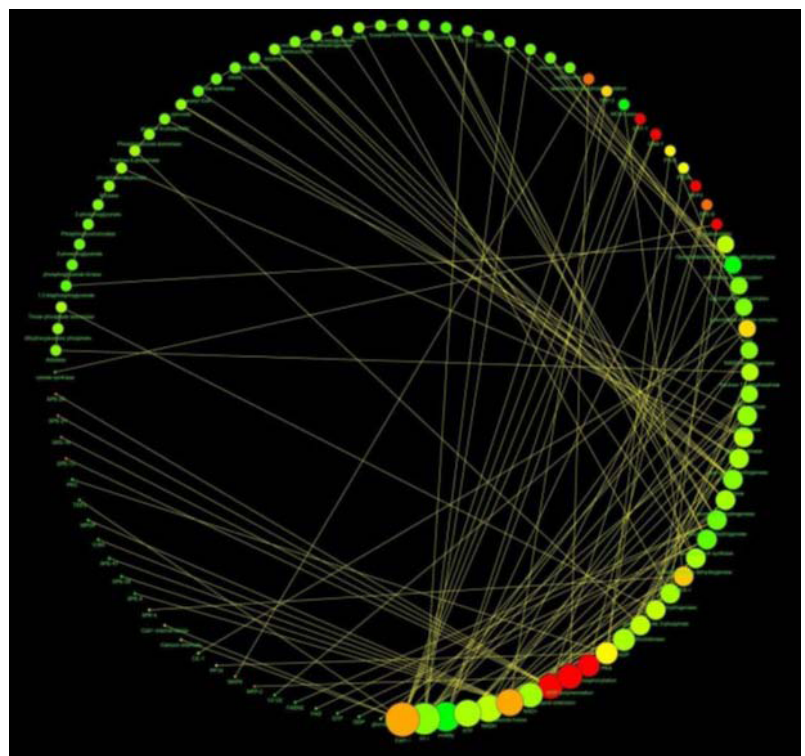

The nodes diameter is proportional to the number of links, the color varies depending on the closeness centrality (see text for explanation). The networks were spatially represented using the Cytoscape Degree Sorted Circle Layout: all nodes with the same number of links are located together around the circle (see Cytoscape User Manual).

Figure 2: Diagram showing the C. elegans spermatozoa activation network.

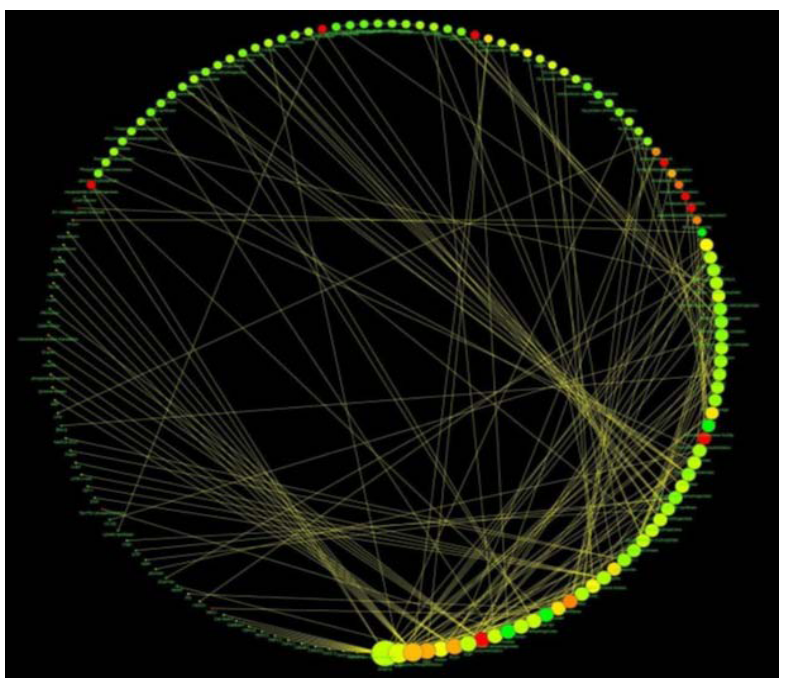

The nodes diameter is proportional to the number of links, the color varies depending on the closeness centrality (see text for explanation). The networks were spatially represented using the Cytoscape Degree Sorted Circle Layout: all nodes with the same number of links are located together around the circle (see Cytoscape User Manual).

Figure 3: Diagram showing the Human spermatozoa activation network.

elegans and Human spermatozoa are showed in Figure 1, 2 and 3 and their main topological parameters are tabulated in Table 1. In all cases the distribution of node linkages follows a power law, represented by the generic equation:

$$
\mathrm{y}=\mathrm{ax}^{-\mathrm{b}}
$$

The $r, \mathrm{R}^{2}$ and $\mathrm{b}$ coefficients of each network are tabulated in Table 2.

The clustering coefficient distribution does not follow a power law, as demonstrated by the results of power law fitting of clustering

\begin{tabular}{|l|c|c|c|}
\hline & Sea urchin & C. elegans & Human \\
\hline $\mathbf{N}^{\circ}$ nodes & 127 & 100 & 151 \\
\hline $\mathbf{N}^{\circ}$ edges & 175 & 132 & 202 \\
\hline $\begin{array}{l}\text { Clustering coef- } \\
\text { ficient }\end{array}$ & 0.023 & 0.032 & 0.028 \\
\hline Diameter & 23 & 23 & 20 \\
\hline Avg. $\mathbf{n}^{\circ}$ neighbours & 2.740 & 2.620 & 2.662 \\
\hline Char. path length & 8.128 & 7.816 & 6.546 \\
\hline
\end{tabular}

The number of nodes represent the total number of molecules involved; the number of edges represents the total number of interactions; the clustering coefficient is calculated as $\mathrm{Cl}=2 n l / k(k-1)$, where $n l$ is the number of links connecting the $\mathrm{kl}$ neighbours of node I to each other; the network diameter is the largest distance between two nodes; the Averaged $n^{\circ}$ neighbours represents the mean number of connections of each node; the Char. path length gives the expected distance between two connected nodes.

Table 1: Main topological parameters of Sea urchin, C. elegans and Human spermatozoa activation networks.

\begin{tabular}{|c|c|c|c|c|c|c|}
\hline & \multicolumn{2}{|c|}{ Sea urchin } & \multicolumn{2}{|c|}{ C. elegans } & \multicolumn{2}{|c|}{ Human } \\
\hline & IN & OUT & IN & OUT & IN & OUT \\
\hline $\mathbf{R}$ & 0.998 & 0.967 & 0.992 & 0.971 & 0.988 & 0.997 \\
\hline $\mathbf{R}^{2}$ & 0.748 & 0.924 & 0.866 & 0.884 & 0.890 & 0.828 \\
\hline b & -1.589 & -2.421 & -2.067 & -2.127 & -1.542 & -1.993 \\
\hline
\end{tabular}

Table 2: Results of power law fitting of IN and OUT sea urchin, C. elegans and Human spermatozoa activation networks.

\begin{tabular}{|l|l|l|l|l|l|}
\hline \multicolumn{2}{|c|}{ Sea urchin } & \multicolumn{3}{c|}{ C. elegans } & \multicolumn{2}{c|}{ Human } \\
\hline Node & $\mathbf{N}^{\circ}$ of links & Node & $\mathbf{N}^{\circ}$ of links & Node & $\mathbf{N}^{\circ}$ of links \\
\hline$\left[\mathrm{Ca}^{2+}\right]_{i}$ & 19 & {$\left[\mathrm{Ca}^{2+}\right]_{i}$} & 10 & {$\left[\mathrm{Ca}^{2+}\right]^{i}$} & 25 \\
\hline$\left[\mathrm{H}^{+}\right]_{i}$ & 14 & {$\left[\mathrm{H}^{+}\right]_{i}$} & 9 & Tyr phosph. & 13 \\
\hline ATP & 9 & ATP & 7 & ATP & 15 \\
\hline cGMP & 15 & Motility & 8 & PKA & 9 \\
\hline cAMP & 13 & Vesicle fusion & 7 & & \\
\hline & & NADH & 7 & & \\
\hline & & 6 & & \\
\hline & & $\begin{array}{l}\text { NAD } \\
\text { Pseudopod exten- } \\
\text { sion }\end{array}$ & 6 & & \\
\hline
\end{tabular}

Table 3: Most connected nodes (the hubs) of sea urchin, C. elegans and Human spermatozoa activation networks.

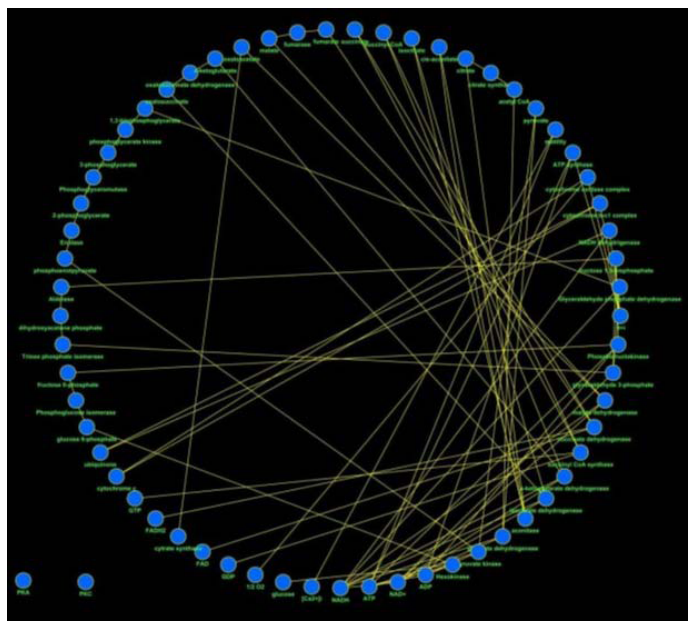

The networks were spatially represented using the Cytoscape Degree Sorted Circle Layout: all nodes with the same number of links are located together around the circle (see Cytoscape User Manual)

Figure 4: Diagram showing the network representing the nodes common to the three networks (CE network).

coefficient distribution $(r=0.598$ in sea urchin; $r=0.100$ in C. elegans; $\mathrm{r}=0.183$ in Human). 


\begin{tabular}{|l|c|}
\hline & CE network \\
\hline $\mathbf{N}^{\circ}$ nodes & 63 \\
\hline $\mathbf{N}^{\circ}$ edges & 80 \\
\hline Clustering coefficient & 0.021 \\
\hline Diameter & 23 \\
\hline Avg. $\mathbf{n}^{\circ}$ neighbours & 2.508 \\
\hline Char. path length & 8.158 \\
\hline
\end{tabular}

The number of nodes represents the total number of molecules involved; the number of edges represents the total number of interactions; the clustering coefficien is calculated as $\mathrm{Cl}=2 n l / k(k-1)$, where $n l$ is the number of links connecting the $k$ neighbours of node I to each other; the network diameter is the largest distance between two nodes; the Averaged $n^{\circ}$ neighbours represents the mean number of connections of each node; the Char. path length gives the expected distance between two connected nodes.

Table 4: Main topological parameters of CE network.

\begin{tabular}{|l|c|c|c|}
\hline & Sea urchin & C. elegans & Human \\
\hline Symmetry & Fivefold & Bilateral & Bilateral \\
\hline Sexes & Male/Female & Hermaphrodite/ Male & Male/Female \\
\hline Fertilization & External & Internal & Internal \\
\hline Sperm motility & Flagellum & Amoeboid & Flagellum \\
\hline Acrosome reaction & Yes & No & Yes \\
\hline Membrane remodeling & No & Yes & Yes \\
\hline Cytoskeleton remodeling & Actin & MSP & Actin \\
\hline Time for sperm activation & Seconds & Days & Hours to days \\
\hline
\end{tabular}

Table 5: Main biological characteristics of reproduction and spermatozoa in sea urchin, C. elegans and Human.

The most connected nodes (the hubs) in the networks (as shown in Table 3) were $\left[\mathrm{Ca}^{2+}\right]_{i}, \mathrm{cGMP},\left[\mathrm{H}^{+}\right]_{\mathfrak{i}}$, aCMP, ATP for sea urchin; $\left[\mathrm{Ca}^{2+}\right]_{\mathfrak{i}},\left[\mathrm{H}^{+}\right]_{\mathfrak{i}}$, Motility, ATP, Vesicle fusion, NADH, NAD ${ }^{+}$, Pseudopod extension for C. elegans; $\left[\mathrm{Ca}^{2+}\right]_{\mathrm{i}}$, ATP, Tyr phosphorylation, PKA for Human.

\section{In all the three networks about the $40-45 \%$ of nodes have two links.}

A new network showing the common elements present in the three networks has been created by computing the intersection of the existing networks (CE network). CE network is represented in Figure 4 and its topological parameters are showed in Table 4 . The nodes belonging to this network are involved in energy metabolism (glicolysis and mitochondrial phosphorylative oxidation) or in signal transduction $\left(\left[\mathrm{H}^{+}\right]_{i}\right.$, PKA, PKC, $\left.\left[\mathrm{Ca}^{2+}\right]_{i}\right)$.

\section{Discussion}

This work was carried out to modelize and compare the signaling machinery of sperm activation processes in organisms belonging to different Phyla such as sea urchin, C. elegans and Human. To this aim, the biological networks representing these events were realized and their main topological parameters were analyzed. This bioinformaticsbased approach is a powerful tool to describe biological organization and function of cellular components as well as to understand the principles driving their evolution [19] and has been already used to represent the biochemical events involved in human [1] and boar [2] spermatozoa activation.

First of all, it was evident that all the networks have virtually the same topological features (see Table 1). This finding is per se really interesting and poses an important question: why so different organisms, living in completely different environments and displaying different biological characteristics (see Table 5) share a common topology for sperm activation?

To answer this question it is necessary to evaluate the biological significance of the networks topological parameters. The most elementary characteristics are the node degree and the clustering coefficient. The first, also known as "connectivity", $k$, indicates how many links the node has to other nodes. It makes possible to define the node degree distribution, $\mathrm{P}(k)$, which represents the probability that a selected node has exactly $\mathrm{k}$ links. The clustering coefficient $\mathrm{CI}=2 n \mathrm{I} / k(k-1)$, where $n \mathrm{I}$ is the number of links connecting the $k \mathrm{I}$ neighbours of node I to each other, measures the network tendency to develop clusters of nodes: more the clustering coefficient is high, more the presence of clusters increases. On the basis of these topological indexes, the networks were classified in: random networks, scale free networks and hierarchical networks. In random networks, described by the Erdös-Rényi (ER) model, the node degrees follows a Poisson distribution, as a consequence the most of nodes have approximately the same number of links (that defines the network's scale) and the clustering coefficient is independent of the nodes degree. The scalefree networks (Barabási- Albert [20], BA, model) are characterized by a power-law degree distribution of the number of links per node: a relatively small number of nodes is highly connected (the hubs) and the most of nodes is scarcely linked, thus it does not exist a "typical" node (scale free topology). In this case the clustering coefficient (CC) is independent of the number of links per node. In hierarchical networks the scale-free topology and the local clustering coexist: the clustering coefficient is higher in the most linked nodes and, consequently, its distribution follows a power law.

The analysis of topological parameters carried out on of sperm activation processes of sea urchin, C. elegans and showed that these networks are preferable to the scale-free networks, as demonstrated by the power law that links the number of edges to the node frequency and the dispersion of clustering coefficient in agreement with the BA model.

From a biological point of view, it is possible to speculate that this particular behavior could offer an important evolutionary advantage: the robustness against random failure. In fact a random perturbation, in most of cases, will involve the most frequent typology of nodes, i.e. the less connected ones, with negligible consequences on network architecture $[1,20,21]$, that is on whole cellular function. In our model the probability that one of them will be involved in random damages is $<5 \%$.

Another interesting finding is the very low values of clustering coefficient which suggest that the architecture of signaling system privileges the rapidity of signal transduction instead the redundancy. Moreover the values of characteristic path length that measures how many links it is necessary to pass through to travel between two nodes, ranging between 6.5 and 8.1 are in line with this hypothesis. These two parameter, kept together with the finding that the higher percentage of nodes display two edges (one input and one output), seem to strengthen the idea that sperm activation is a highly efficient signaling process. In fact:

each node receives an input signal and transfers an output response;

any molecule can interact with any other in a small number of passages, thus, the loss of information due to the signal decrease is minimized and the signal efficiency is maximized;

any local perturbation in signaling system could reach the whole network in a short time increasing the system responsiveness to intracellular and extracellular stimuli. 
In the same context it is important to point out as the terminal events (such as "protein phosphorylation" or "membrane fusion" or "motility") are highly linked. Reasonably this is due to the redundancy of biochemical signaling, as a safety strategy to overlap partial failure of the system. The most linked node was, in all the cases, the same: $\left[\mathrm{Ca}^{2+}\right]_{i}$. This finding is not surprising, in fact, as in many other cellular systems, in spermatozoa activation $\mathrm{Ca}^{2+}$ behaves as a second messenger.

More in detail in sea urchin the $\mathrm{Ca}^{2+}$ enters the sperm cell through voltage dependent channels (Cav1.2 or 1.3) or through cAMP or cGMP (SpHCN1 or 2) gated channels. In the intracellular signalling cascade this ion is involved in the control of cAMP, cGMP and in the PIP2/ IP3 pathway [22]. In C. elegans $\left[\mathrm{Ca}^{2+}\right]_{i}$ is controlled by membrane channels and by sequestering the ion in intracellular stores and it is involved in PIP2/IP3 signaling pathways regulating the MOs fusion and the onset of motility [9]. In Human it is known that during capacitation the $\left[\mathrm{Ca}^{2+}\right]_{\mathrm{i}}$ increases and the capacitation does not take place in $\mathrm{Ca}^{2+}$ absence $[10,23]$. Four major $\mathrm{Ca}^{2+}$ clearance mechanisms are described in mammalian spermatozoa, two acting on the plasma membrane and two acting on intracellular organelles. The plasma membrane $\mathrm{Ca}^{2+}$-ATPase exports a cytoplasmic $\mathrm{Ca}^{2+}$ ion and imports one or two extracellular protons at the expense of ATP. When $\left[\mathrm{Ca}^{2+}\right]$ is elevated, the plasma membrane $\mathrm{Na}^{+}-\mathrm{Ca}^{2+}$ exchanger operates in forward mode exporting an intracellular $\mathrm{Ca}^{2+}$ ion and importing approximately three $\mathrm{Na}^{+}$ions at the expense of the $\mathrm{Na}^{+}$gradient $[24,25]$. The best characterized organellar clearance mechanism involves the sarcoplasmic-endoplasmic reticulum $\mathrm{Ca}^{2+}$-ATPase pumps and the mitochondrial $\mathrm{Ca}^{2+}$ uniporter [25]. During the sperm capacitation the $\mathrm{Ca}^{2+}$ acts converting extracelluar stimuli to chemical response in a myriad of molecular system, such as, protein kynase $\mathrm{C}$ (PKC), protein kynase A (PKA), actin, and many others.

The other most connected nodes belong to different classes:

- Molecules involved in energetic balance, such as ATP. It is the main energetic source for spermatozoa, where the metabolic energy production derives exclusively from the glycolysis and from mitochondrial oxidative phosphorylation [26].

- $\left[\mathrm{H}^{+}\right]_{\mathrm{i}}$ : in sea urchin and C. elegans the $\mathrm{pH}$ is a key element in control of many biochemical events, such as the membrane polarization, the rearrangement of cytoskeleton proteins (MSP) and the motility $[9,27]$. It is important to note that in Human and mammals spermatozoa the $\mathrm{pH}$ modification is an early event in the acquisition of full fertilizing ability [13].

- Molecules playing a key role in signal transduction and amplification: cAMP, cGMP. These two cyclic nucleotides are ubiquitous second messengers and concur to the control of cellular signal transduction by modulating the activity of membrane channels, kinases, phosphatases and many other molecules.

- Complex events whose biochemical determinants are not completely known ("Tyr phosphorylation" in Human, "Motility", "Pseudopod extension", "Vesicle fusion" in C.elegans).

The analysis of topological parameters of the CE networks, showed that the elements common to the three networks are those representing the scaffold of spermatozoa energetic metabolism (in all the cases the energy is provided, as ATP, by glicolysis and mitochondrial phosphorylative oxidation) or the signaling system $\left[\mathrm{Ca}^{2+}\right]_{i},\left[\mathrm{H}^{+}\right]_{\text {, }}$ PKA, PKC.
From these data, it is evident that spermatozoa activation in sea urchin, C. elegans and Human recognizes similar biochemical determinants. This finding strengths that some signaling molecules and metabolic processes (such as $\left[\mathrm{Ca}^{2+}\right]_{i}$, glycolysis, mitochondrial oxidative phosphorylation) have an ubiquitous role in spermatozoa signaling and biochemistry; in this context it will be interesting to compare the studied networks with the data concerning different cellular systems undergoing an activation process (for instance neurons, leucocytes, myocardiocytes)

More interesting is the evidence that the networks topology of the three networks is virtually identical displaying important features:

- robustness against random failure;

- signaling fastness, rapidity and efficiency.

Since, as Dobzhansky [28] famously stated, "nothing in biology makes sense except in the light of evolution", it is possible to hypothesize that the similarity in the architecture of this process in such different organisms could be due to the maintenance of similar ancestral mechanisms that offer important evolutionary advantages.

In our opinion these findings in one hand strength the usefulness of bioinformatics modeling in the study of the biochemical asset of different organisms from a comparative and evolutionary point of view, in the other hand could contribute to the knowledge of the spermatozoa activation, i.e. of a process of pivotal importance for the survival of living beings.

\section{References}

1. Bernabò N, Mattioli M, Barboni B (2010) The spermatozoa caught in the net: the biological networks to study the male gametes post-ejaculatory life. BMC Syst Biol 4: 87 .

2. Bernabò N, Berardinelli P, Mauro A, Russo V, Lucidi P et al. (2011) The role of actin in capacitation-related signaling: an in silico and in vitro study. BMC Syst Biol 5: 47

3. Barnes RD (1992) Invertebrate Zoology. Holt-Saunders International edition Tokio.

4. Neill AT, Vacquier VD (2004) Ligands and receptors mediating signal transduction in sea urchin spermatozoa. Reproduction 127: 141-149.

5. Nishigaki T, Chiba K, Miki W, Hoshi M (1996) Structure and function of asterosaps, sperm- activating peptides from the jelly coat of starfish eggs. Zygote 4: 237-245

6. Barré P, Zschornig O, Arnold K, Huster D (2003) Structural and dynamical changes of the bindin B18 peptide upon binding to lipid membranes. A solidstate NMR study. Biochemistry 42: 8377-83786.

7. Kamei N, Glabe CG (2003) The species-specific egg receptor for sea urchin sperm adhesion is EBR1, a novel ADAMTS protein. Genes Dev 17: 2502-2507.

8. L'Hernault SW (2006) Spermatogenesis. WormBook 20: 1-14

9. Fraire-Zamora JJ, Cardullo RA (2010) The Physiological Acquisition of Amoeboid Motility in Nematode Sperm: Is the Tail the Only Thing the Sperm Lost? Mol Repr Dev 77: 739-750.

10. Breitbart H (2002) Intracellular calcium regulation in sperm capacitation and acrosomal reaction. Mol Cell Endocrinol 187: 139-144.

11. Boni R, Gualtieri R, Talevi R, Tosti E (2007) Calcium and other ion dynamics during gamete maturation and fertilization. Theriogenology 68: 156-164.

12. Barbonetti A, Vassallo MR, Cinque B, Antonangelo C, Sciarretta $F$ et al (2008) Dynamics of the global tyrosine phosphorylation during capacitation and acquisition of the ability to fuse with oocytes in human spermatozoa. Biol Reprod 79: 649-656

13. Abou-haila A, Tulsiani DR (2009) Signal transduction pathways that regulate sperm capacitation and the acrosome reaction. Arch Biochem Biophys 485: 72-81. 
Citation: Bernabò N, Saponaro I, Mattioli M, Barboni B (2012) TSignaling Strategy in Spermatozoa Activation of Sea Urchin, C. elegans and Human: Three Different Players for the Same Melody. J Bioengineer \& Biomedical Sci S5:006. doi:10.4172/2155-9538.S5-006

Page 6 of 6

14. Brener E, Rubinstein S, Cohen G, Shternall K, Rivlin J, et al. (2003) Remodeling of the actin cytoskeleton during mammalian sperm capacitation and acrosome reaction. Biol Reprod 68: 837-845

15. Gadella BM, Tsai PS, Boerke A, Brewis IA (2008) Sperm head membrane reorganization during capacitation. Int J Dev Biol 52: 473-480.

16. Botto L, Bernabò N, Palestini P, Barboni B (2010) Bicarbonate induces membrane reorganization and CBR1 and TRPV1 endocannabinoid receptor migration in lipid microdomains in capacitating boar spermatozoa. $\mathrm{J}$ Membr Biol 238: 33-41.

17. Suarez SS (2008) Control of hyperactivation in sperm. Hum Reprod Update 14: $647-657$

18. Bollobas B (1979) Graph Theory: an Introductory Course. New York: Springer Verlag.

19. Vespignani A (2003) Evolution thinks modular. Nat Genet 35: 118-119.

20. Albert R, Jeong H, Barabasi AL (2000) Error and attack tolerance of complex networks. Nature 406: 378-382
21. Barabasi AL (2001) The physics of the Web. Physics World 14: 33-38.

22. Darszon A, Nishigaki T, Wood C, Trevino CL, Felix R, et al. (2005) Calcium channels and $\mathrm{Ca}^{2+}$ fluctuations in sperm physiology. Int Rev Cytol 243: 79-172.

23. Florman HM, Jungnickel MK, Sutton KA (2008) Regulating the acrosome reaction. Int J Dev Biol 52: 503-510.

24. Blaustein MP, Lederer WJ (1999) Sodium/calcium exchange: its physiological implications. Physiol Rev 79: 763-854.

25. Wennemuth G, Babcock BF, Hille B (2003) Calcium Clearance Mechanisms of Mouse Sperm. J Gen Physiol 122: 115-128.

26. Storey BT (2008) Mammalian sperm metabolism: oxygen and sugar, friend and foe. Int J Dev Biol 52: 427-437.

27. Solzin J, Helbig A, Van Q, Brown JE, Hildebrand E, et al. (2004) Revisiting the role of $\mathrm{H}+$ in chemotactic signaling of sperm. J Gen Physiol 124: 115-124.

28. Dobzhansky T (1973) Nothing in biology makes sense except in light of evolution. Am Biol Teacher 35: 125-129. 